\title{
On general curves lying on a quadric.
}

\author{
By Professor H. F. Baker, F.R.S. \\ (Received 7th September 1926. Read 5th November 1926.)
}

Introduction. The present note, though in continuation of the preceding one dealing with rational curves, ${ }^{1}$ is written so as to be independent of this. It is concerned to prove that if a curve of order $n$, and genus $p$, with $k$ cusps, or stationary points, lying on a quadric, $\Omega$, in space of any number of dimensions, is such that itself, its tangents, its osculating planes, $\ldots$, and finally its osculating $(h-1)$-folds, all lie on the quadric $\Omega$, then the number of its osculating $h$-folds which lie on the quadric is

$$
2[n+2 h(p-1)]-2 k .
$$

Two proofs of this result are given, in $\S \S 4$ and 5 .

For $p=0$, that is for a rational curve, this result is incidentally proved in the preceding note. For $h=1$ the result, as will be seen, is well known; and it may well be that the general result has been stated. In any case the note suggests a further enquiry, to the case when, in place of a curve, a locus of points, the locus of an aggregate of lines, or of planes, etc., is considered.

§1. Consider a Ruled surface in ordinary space. It possesses a certain number of generators which intersect their "consecutive." Let the general generator be represented, in Cartesian coordinates $x, y, z, \mathrm{by}$

$$
x=\xi+z a, y=\eta+z \beta,
$$

where $(\xi, \eta, 0)$ is a general point of the section by $z=0$, and $\alpha, \beta, \gamma$ are regarded as functions of $\xi$, the points of the generator being given by the various values of $z$. The condition for this generator to intersect its consecutive may be obtained by expressing that the point of intersection $(x, y, z)$, must also satisfy the equations

$$
1+z \frac{d \alpha}{d \xi}=0, \frac{d \eta}{d \bar{\xi}}+z \frac{d \beta}{d \xi}=0
$$

${ }^{1}$ Proc. Edin. Math. Sor., XLIV, 1926, p. 131. 
the generators in question arise therefore for values of $\xi$ such that

$$
\frac{d \eta}{d \xi} \frac{d \alpha}{d \xi}-\frac{d \beta}{d \xi}=0
$$

The tangent plane of the ruled surface is then the same at every point of such a generator, its equation being

$$
y-\eta-\beta z-(x-\xi-\alpha z) \frac{d \eta}{d \bar{\xi}}=0,
$$

and every plane through the generator touches the surface at the point of the generator for which

$$
z=-\left(\frac{d \alpha}{d \xi}\right)^{-1}
$$

Conversely these facts may be used to define the generator, which is hence called a Torsal line.

The number of such generators may be found by the theorem (Salmon, Solid Geometry, 1882, p. 591) that the Hessian of the Ruled surface meets the surface in its double curve, counting eight times, and in the Torsal lines, each counting twice. The order of the plane section being $n$, and its genus $p$, so that, if we neglect the possibility of cuspidal generators, the order of the double curve is

the equation

$$
\frac{1}{2}(n-1)(n-2)-p,
$$

$$
8\left[\frac{1}{2}(n-1)(n-2)-p\right]+2 x=4 n(n-2)
$$

gives for $x$, the number of Torsal lines,

$$
x=2(n+2 p-2) .
$$

Another proof of this number is obtained by considering two plane sections of the Ruled surface, put in $(1,1)$ correspondence by the generators, and finding the number of intersections of a tangent of one section with the tangent at the corresponding point of the other.

We easily find that the line coordinates of the general generator of the Ruled surface are

$$
l=\alpha, m=\beta, n=1, l^{\prime}=\eta, m^{\prime}=-\xi, n^{\prime}=\xi \beta-\eta \alpha,
$$

so that, when this is a Torsal line, we have

$$
d l d l^{\prime}+d m d m^{\prime}+d n d n^{\prime}=\mathbf{0}
$$


if $\left(l, m, n, l^{\prime}, m^{\prime}, n^{\prime}\right)$ be regarded as the coordinates of a point in space of five dimensions, thus lying on the quadric, $\Omega$, given by

$$
l l^{\prime}+m m^{\prime}+n n^{\prime}=0
$$

the generators of the Ruled surface will be represented by the points of a curve lying on $\Omega$. Those particular generators which are torsal will be represented by points of this curve whereat the tangent line of the curve lies wholly on $\Omega$. This is what is expressed by the differential equation $d l d l^{\prime}+\ldots=0$, which is equivalent to the original differential equation $d \eta / d \xi-d \beta / d a=0$.

$\S 2$. It is obvious that two lines of ordinary space which intersect determine a flat pencil of lines; thus, if the lines of ordinary space be represented by the points of a quadric, $\Omega$, in space of five dimensions, the line joining the points of this quadric which represent two intersecting lines of ordinary space lies wholly on $\Omega$. Thus the generating lines of a developable surface of ordinary space are represented in five dimensions by the points of a curve, lying on $\Omega$, which has the property that all its tangents equally lie on $\Omega$. A ruled surface which is not a developable will however similarly be represented by a curve on $\Omega$ of which the tangents at the points representing the torsal generators of the ruled surface lie on $\Omega$.

The order of the curve on $\Omega$ which represents a ruled surface of ordinary space, being, by definition, the number of its intersections with a general prime (or fourfold) of the fivefold space, is equal to the number of its intersections with a tangent prime of $\Omega$; that is, is equal to the number of generators of the ruled surface which meet any general line, that is, to the order of the ruled surface. By the $(1,1)$ correspondence, the genus of the representative curve on $\Omega$ is equal to the genus of the ruled surface, or of an arbitrary plane section of this surface.

In the particular case when the ruled surface is the developable formed by the tangents of a curve, $C$, of ordinary space, the order of the representative curve, on $\Omega$, is the rank, $r$, of the curve $C$, the number of its tangents meeting a general line. Further, the points of the curve $C$ are represented on $\Omega$ by the planes of the first kind lying on $\Omega$, say the planes (I), which pass through the tangent lines of the representative curve on $\Omega$, while the osculating planes of the curve $C$ are represented by the planes of the second kind lying on $\Omega$, say the planes (II), which pass through the tangent lines of the 
representative curve on $\Omega$. Thus the order, $n$, of the curve $C$, being the number of its points lying on an arbitrary plane, is the number of planes (I), on $\Omega$, through the tangent lines of the representative curve, which meet any selected plane (II) of $\Omega$ in a line, or, is the number of tangent lines of the representative curve (say $\gamma$ ) which meet this plane (II). Similarly, the class, $n^{\prime}$, of the curve $C$, being the number of osculating planes through a general point, is the number of tangent lines of the curve $\gamma$, on $\Omega$, which meet any selected plane $(I)$, of $\Omega$. If the curve $C$ have a point of inflexion, there being three "consecutive" points of the curve, or three "consecutive" osculating planes, lying in, or through, the same tangent line, that is, two "consecutive" tangent lines which are the same line, then the representative curve has a stationary point or cusp; let $\beta$ be the number of inflexions of $C$, or cusps of $\gamma$. Consider now the number of tangent lines of the curve $\gamma$, on $\Omega$, which meet an arbitrary solid (threefold space) of the fivefold space; such lines will lie in a prime (fourfold space) through this solid; conversely if any prime be drawn through this solid, the joining line of any two of the $r$ intersections of the prime with the curve $\gamma$ will meet the solid, and if the two intersections coincide, except at a cusp, the line will be a tangent line which meets the solid. The primes through the solid are a single infinity, ("pencil"), and determine an $\infty^{1}$ lot of sets of $r$ points upon the curve $\gamma$, of genus $p$. There are, therefore, by a result originally due to Riemann, $2(r+p-1)$ cases of coincidence of two points of such a set, including once every cusp of the curve $\gamma$, or so many tangent lines meeting the fixed solid, if the $\beta$ cusps be reckoned in. Let us, in particular, take for the fixed solid one determined by a plane (I) of $\Omega$, together with a plane (II) which meets the former in a line; this solid is the polar solid, in regard to $\Omega$, of the line of intersection of these two planes and intersects $\Omega$ only in these two planes. The tangent lines of the curve $\gamma$, which lie on $\Omega$, can only meet this solid on the plane (I), or the plane (II). As we have seen, the total number of such meetings is $n+n^{\prime}$. Allowing for the cusps, $\beta$ in number, we thus have the result

$$
n+n^{\prime}+\beta=2(r+p-1),
$$

which is a known result of the Plücker-Cayley formulae for the curve $C$. Another result of these formulae, $r+\alpha=2 n+2 p-2$, may be similarly illustrated. Here, $\alpha$ is the number of stationary points, or cusps, of the curve $C$ itself, the number of "points in four planes" 
(Salmon, Solid Geometry, 1882, p. 292; there denoted by $\beta$ ); in other words $\alpha$ is the number of points of the curve $C$ through which three, instead of two, "consecutive" tangents of the curve pass; for the curve $\gamma$ it is therefore the number of points at which the osculating plane lies on $\Omega$, and is a plane (I) of $\Omega$. Similarly, the number $\alpha^{\prime}$, of stationary planes of the curve $C$, is the number of osculating planes of the curve $\gamma$ which are planes (II) of $\Omega$. And another result of the Plucker-Cayley formulae for the curve $C$, namely

$$
a+\alpha^{\prime}+2 \beta=2(r+4 p-4),
$$

is thus also capable of interpretation. For we shall prove below that the right side, for a curve lying on a quadric, of order $r$ and genus $p$, of which the tangent lines lie on the quadric, is the number of osculating planes of the curve which lie on the quadric. We have already seen this when $p=0$.

§3. The results so far suggested to us for a curve upon a quadric in five dimensioned space which arises as representing a ruled surface of ordinary space, are in fact true for a curve lying on a quadric in space of any number of dimensions. We first illustrate this by obtaining again, in another manner, the formula which, in the particular case we have considered, gives the number of torsal generators of a ruled surface.

We consider, in space of any number of dimensions, a quadric, $\Omega$, given by the vanishing of a single quadratic polynomial in the coordinates; and, upon this quadric, a curve, $C$, of order $n$ and genus $p$. We shew that the number of points of this curve whereat the tangent line lies on the quadric is $2(n+2 p-2)-2 k$, where $k$ is the number of stationary points, or cusps, of the curve.

The tangent prime of the quadric at any ordinary point, $P$, of the curve, meets the curve again in $(n-2)$ points. The line joining $P$ to one of these points, $P^{\prime}$, meets the quadric in two coincident points at $P$, and thus lies entirely on the quadric, so that the tangent prime of the quadric at $P^{\prime}$ contains $P$. We thus have a correspondence, of indices $(n-2, n-2)$, between two points $P, P^{\prime}$ of the curve whose join lies on the quadric. This correspondence is of valency 2 , since, obviously, the $(n-2)$ points corresponding to $P$, together with $P$ taken twice over, are the complete intersection of the curve with the tangent prime at $P$, and are thus a set of the linear series $\left(\infty^{1}\right)$ of sets of $n$ points in which the curve is met by the 
primes of the space. Thus, by the Cayley-Brill formula, there are $2(n-2)+2.2 p$, or $2(n+2 p-2)$,

cases in which $P$ coincides with one of the $(n-2)$ corresponding points. When this is so the chord becomes the tangent of the curve at $P$ and lies entirely on the quadric, if $P$ be an ordinary point of the curve. But, it may be shewn, the number obtained contains every cusp of the curve, counted twice; this appears more clearly from the second proof of the theorem given below in $\S 5$.

For example, if the quadric $\Omega$ (and the curve $C$ ) be in space of three dimensions, and the curve be a cubic, for which $n=3, p=0$, the number obtained is 2 . This we know to be accurate; for the generators of the quadric which meet the cubic curve in two points determine an involution on this curve, of which there are two double points. If the curve $C$ be a quartic with $p=1$, the intersection of the quadric with another quadric, the number obtained is 8 . This again is a familiar result, there being four generators of each system of the original quadric which touch the curve, as we may see by projecting the curve, and one system of generators, from one of the vertices of the self polar tetrahedron, two quadric cones of common vertex having four common tangent planes. If the curve be a nonsingular rational quartic, part of the intersection of the given quadric with a cubic surface containing two skew generators of the quadric, the number obtained is 4; namely, of the generators of that system of the quadric which are trisecant chords of the curve, there are four which touch the curve. This is also obvious by remarking that, if two points of the curve be regarded as corresponding when they lie on a trisecant chord, this establishes a $(2,2)$ correspondence, of which there are four points of coincidence. The curve may however be the rational intersection of two quadrics which touch, having a double point, or a cusp at this point. In the former case the number $2(n-2)$, or 4 , is still the right number of tangents of the curve which lie on either quadric, there being two generators of each system, of the quadric, which are tangent lines of the curve. In the latter case the number $2(n-2)-2 k$, or 2 , is also correct, there being only one generator of each system, of the quadric, touching the curve. This may be verified in detail by taking the two quadrics to be

$$
z t=x^{2}-y^{2}, \quad z t=(a+1) x^{2}+(b-1) y^{2}+z(l x+m y) ;
$$

these have contact at $x=y=z=0$, which is stationary if $a$ or $b$ is zero. 
For the case when the quadric $\Omega$ is in space of five dimensions, and the $C$ curve represents a ruled surface of ordinary space, we have already shewn that a point of $C$ where the tangent line lies on $\Omega$ corresponds to a torsal generator of the ruled surface. The number of these is then $2(n+2 p-2)$, as we have already found, this number including however every stationary generator of the ruled surface, counted twice.

$\S 4$. It may happen that of the curve $C$ on $\Omega$ every tangent also lies on $\Omega$. We may then investigate how many of the osculating planes of $C$ lie on $\Omega$. It will be as easy, however, at least in the former of the two methods which we employ, to suppose that, of the curve $C$, every osculating $(h-1)$-fold-determined by $h$ "consecutive" points of $C$-lies on $\Omega$, and to investigate how many osculating $h$-folds of $C$ lie on $\Omega$. We prove that this number is

$$
2[n+2 h(p-1)]-2 k,
$$

where $k$ is the number of cusps of $C$.

The proof is quite easy if we assume the following lemma, of which we give the proof in outline in $\$ 6$ below:-

When the curve $C$ is such that every osculating $(h-1)$-fold, determined by $h$ "consecutive" points of the curve, lies on the quadric $\Omega$, then the tangent prime of $\Omega$ at any ordinary point, $P$, of the curve, meets the curve at $P$ in $2 h$ coincident points. But if, for a particular ordinary point of the curve, the tangent prime of $\Omega$ contains $2 h+1$ coincident points of the curve, then the osculating $h$-fold of the curve at that point lies entirely on the quadric $\Omega$.

There is limitation ${ }^{1}$ of the value of $h$ owing to the fact that upon a quadric in space of $r$ dimensions, the linear space of highest dimension, $s$, must be such that $s<\frac{1}{2} r$. We shall suppose that $h<\frac{1}{2} r$, if the curve, and $\Omega$, be in space of $r$ dimensions.

Assuming this lemma, and supposing that every osculating $(h-1)$-fold of the curve $C$ lies on $\Omega$, the tangent prime of $\Omega$, at any ordinary point, $P$, of $C$, meets the curve again in $n-2 h$ points.

1 For instance, if we can have a rational quintic curve, with its tangents, lying upon a quadric in space of four dimensions $(n=5, h=2, r=4)$, the result of the formula would otherwise be that there are two of its osculating planes upon the quadric. In the preceding paper, dealing with rational curves, we have supposed the curve of order $n$ to lie in space of $n$ dimensions. 
Thus we have a $(n-2 h, n-2 h)$ correspondence between pairs of points of this curve, evidently of ralency $2 h$. There will then be, neglecting the possibility of cusps,

$$
2(n-2 h)+2.2 h \cdot p,=2[n+2 h(p-1)],
$$

coincidences, or, this number of points $P$ where, by the lemma, the osculating $h$-fold of $C$ lies on $\Omega$; which is the theorem stated. The correction when $C$ has cusps appears clearly in the second proof of the theorem given in $\$ 5$ below.

For instance, when $h=2$, that is, for a curve on $\Omega$ of which every tangent line lies on $\Omega$, the number of points of $C$ for which the osculating plane also lies on $\Omega$ is

$$
2(n+4 p-4),
$$

as was remarked above for the case when $\Omega$ is in five dimensions.

$\S 5$. There is another method of proof of this result, which is instructive, proceeding with a step by step process.

Suppose as before the curve $C$ to lie on $\Omega$, a quadric in space of $r$ dimensions, and that we wish to find how many of its tangents lie on ऽ. From a general point $O$, of $\Omega$, let $\Omega$, and the curve $C$, be projected upon a space $\bar{\sigma}$, of $r-1$ dimensions. The tangent prime of $\Omega$ at $O$ contains all lines through $O$ which lie on $\Omega$; the tangent prime meets $\sigma$ in a space, $\sigma^{\prime}$, of $r-2$ dimensions, and these lines determine therein a quadric, $\Omega^{\prime}$, of dimension $r-3$. The curve $C$ meets the tangent prime of $\Omega$ at $O$ in $n$ points; the curve $C^{\prime}$, in $\pi$, which is the projection of $C$, thus meets the quadric $\Omega^{\prime}$ in $n$ points, these being the intersections of $C^{\prime}$ with the space $\sigma^{\prime}$. Any tangent line of $C$ meets the tangent prime of $\Omega$ at $O$ in a point, and is thus projected into a tangent line of $C^{\prime}$ meeting $\sigma^{\prime}$. But a tangent line of $C$ which lies on $\Omega$ meets the tangent prime of $\Omega$ at $O$ in a point on $\Omega$, and is thus projected into a tangent line of $C^{\prime \prime}$ which meets $\sigma^{\prime}$ in a point of $\Omega^{\prime}$. To find the tangent lines of $C$ which lie on $\Omega$, we are thus required to find the intersections with $\Omega^{\prime}$ of the curve, $\gamma$, in $\bar{\sigma}^{\prime}$, which is the locus of the intersections, with $\sigma^{\prime}$, of the tangents of $C^{\prime}$. The number of these intersections would be twice the order of this curve $\gamma$, were it not for the $n$ intersections of $C^{\prime}$ itself with $\Omega^{\prime}$; at such an intersection there are two coincident tangents of $C^{\prime}$ meeting $\Omega^{\prime}$, and these do not generally arise by projection from tangents of $C$ which lie on $\Omega$ (the pusition of $O$ being arbitrary). Now, the order of the curve $\gamma$ is the 
number of tangents of the curve $C^{\prime}$ which meet a space $S_{r-3}$, of dimension $(r-3)$; or is the number of $S_{r_{2}}$, of the pencil of such $S_{r-2}$ which pass through this $S_{r-3}$, which touch the curve $C^{\prime}$, which itself lies in the prime $\varpi$, of dimension $(r-1)$. If $C$ have $k$ cusps, and therefore as $O$ is arbitrary, if $C^{\prime}$ have $k$ cusps, this number is $2 n+2 p-2-k$, by a theorem originally due to Riemann. The number of tangents of $C$ which lie on $\Omega$ is thus

or

$$
\begin{gathered}
2(2 n+2 p-2-k)-2 n \\
2(n+2 p-2)-2 k
\end{gathered}
$$

as was remarked.

Next suppose that all the tangent lines of $C$ lie upon $\Omega$, and that we wish to find how many osculating planes of $C$ lie on $\Omega$. Carrying out the same projection, the curve $\gamma$ will be entirely upon $\Omega^{\prime}$. An osculating plane of $C$ will give rise by projection to a tangent line of the curve $\gamma$, for the osculating plane meets the tangent prime of $\Omega$ at $O$ in a line. If this osculating plane lies on $\Omega$, this line also lies on $\Omega$, and the corresponding tangent line of $\gamma$ will be on $\Omega^{\prime}$. If $\nu$ be the order of $\gamma$, the number of such tangents, by what has been seen, is

$$
2(\nu+2 p-2)-2 k^{\prime},
$$

where $k^{\prime}$ is the number of cusps of $\gamma$. These cusps are the $n$ points of $\gamma$ where $C^{\prime}$ meets $\Omega^{\prime}$. The number of osculating planes of $C$ which lie on $\Omega$, putting

is thus

$$
\nu=2 n+2 p-2-k, k^{\prime}=n,
$$

$$
2(n+4 p-4)-2 k .
$$

Next suppose the osculating planes of $C$ lie on $\Omega$, and we wish to find the number of osculating solids (threefolds) of $C$ which lie on $\Omega$. This will as before be the number of osculating planes of $\gamma$ which lie on $\Omega^{\prime}$, and will therefore be

or

$$
\begin{aligned}
& 2(\nu+4 p-4)-2 k^{\prime}, \\
& 2(n+6 p-6)-2 k .
\end{aligned}
$$

The argument is evidently general, and we have the same result as before

$\S 6$. We may now indicate the nature of an elementary proof of the lemma assumed in $\S 4$.

The equation of $\Omega$ may be taken to be

$$
x_{1} y_{1}+x_{2} y_{2}+\ldots+x_{k} y_{k}+m x_{k+1}^{2}=0,
$$


where $m$ is a constant, equal to zero when the space in which $\Omega$ lies is of odd dimension $(2 k-1)$, and equal to unity when the space is of even dimension $(2 k)$. In the latter case we can write the last term $x_{k+1} y_{k+1}$ with $y_{k+1}=x_{k+1}$. This form, an evident change from a sum of $2 k$ (or $2 k+1$ ) squares, is always possible.

The quadric contains the point for which

$$
x_{1}=y_{1}=\ldots=x_{k}=0 \text { and } x_{k+1}=0, \text { with } y_{k}=1 \text {, }
$$

and the tangent prime at this point is $x_{k}=0$. We suppose the curve on $\Omega$, which we consider, to pass through this point, and to have, for the expression of its coordinates, in terms of a parameter $s$, in this neighbourhood, forms indicated by

$$
\begin{aligned}
x_{1} & =\frac{a_{1}{ }^{(1)}}{1 !} s+\frac{a_{2}{ }^{(1)}}{2 !} s^{2}+\ldots \quad, y_{1}=\frac{b_{1}{ }^{(1)}}{1 !} s+\frac{b_{2}{ }^{(1)}}{2 !} s^{2}+\ldots, \\
x_{k-1} & =\frac{a_{1}^{(k-1)}}{1 !} s+\frac{a_{2}^{(k-1)}}{2 !} s^{2}+\ldots, \quad y_{k-1}=\frac{b_{1}{ }^{(k-1)}}{1 !} s+\frac{b_{2}{ }^{(k-1)}}{2 !} s^{2}+\ldots, \\
y_{k+1}= & =x_{k+1},=\frac{a_{1}{ }^{(k+1)}}{1 !} s+\frac{a_{2}{ }^{(k+1)}}{2 !} s^{2}+\ldots,
\end{aligned}
$$

together with

$$
x_{k}=\frac{p_{1}}{1 !} s+\frac{p_{2}}{2 !} s^{2}+\ldots, \quad y_{k}=1,
$$

where the denominators are factorials.

The conditions that the curve lies on $\Omega$ require that if these series be substituted in the equation of the quadric $\Omega$, the coefficient of every power of $s$ in the resulting series should vanish. Instead now of writing separately the results of the substitution of these series in the terms

$$
x_{1} y_{1}, x_{2} y_{2}, \ldots, x_{k-1} y_{k-1}, m x^{2}{ }_{k+1},
$$

preceded by a sign of summation over the $k$ terms (in case the last term is present), we shall write down simply the result of substituting

$$
x=\frac{a_{1}}{1 !} s+\frac{a_{2}}{2 !} s^{2}+\ldots, y=\frac{b_{1}}{1 !} s+\frac{b_{2}}{2 !} s^{2}+\ldots,
$$

in the single term $x y$. It is then understood that the coefficient of every power of $s$ in the result of the substitution stands for the sum of the results of the substitutions in these $k$ terms (or $k-1$ terms when $m=0$ ). Further we shall denote $a_{p} b_{q}+a_{r_{l}} b_{\rho}$ by $[p, q]$, 
and $a_{p} b_{p}$ by $[p, p]$; also, the product of the two factorials $(p)$ ! and $(q) !$, that is $1.2 \ldots p .1 .2 \ldots q$ may be denoted by $(p . q)$.

The condition that the curve lies on the quadric is thus represented by the vanishing of

$$
\frac{[1,1]}{(1.1)} s^{2}+\frac{[1,2]}{(1.2)} s^{3}+\left(\frac{[1,3]}{(1.3)}+\frac{[2,2]}{(2.2)}\right) s^{4}+\ldots+\frac{p_{1}}{1 !} s+\frac{p_{2}}{2 !} s^{2}+\ldots=0,
$$

requiring the conditions

$p_{1}=0, \frac{[1,1]}{(1.1)}+\frac{p_{2}}{2 !}=0, \frac{[1,2]}{(1.2)}+\frac{p_{3}}{3 !}=0, \frac{[1,3]}{(1.3)}+\frac{[2,2]}{(2.2)}+\frac{p_{4}}{4 !}=0$, etc.

The conditions that the tangents of the curve lie on the quadric are found (as is easy to see) by adding to these conditions those obtained by substituting for the coordinates $x, y$ the series for $\frac{d x}{d s}, \frac{d y}{d s}$, or say $x^{\prime}, y^{\prime}$, namely

$$
x^{\prime}=\frac{a_{1}}{0 !}+\frac{a_{2}}{1 !} s+\frac{a_{3}}{2 !} s^{2}+\ldots, y^{\prime}=\frac{b_{1}}{0 !}+\frac{b_{2}}{1 !} s+\frac{b_{3}}{2 !} s^{2}+\ldots,
$$

and for $x_{k}$ the series

$$
x_{k}^{\prime}=\frac{p_{1}}{0 !}+\frac{p_{2}}{1 !} s+\frac{p_{3}}{2 !} s^{2}+\ldots
$$

but as $d y_{k} / d s=0$, the series for $x_{k}^{\prime}$ does not arise in this substitution. The result of the substitution is

$$
\frac{[1,1]}{(0.0)}+\frac{[1,2]}{(1.1)} s+\left(\frac{[1,3]}{(1.2)}+\frac{[2,2]}{(1.1)}\right) s^{2}+\ldots=0
$$

and leads to

$$
\frac{[1,1]}{(0.0)}=0, \frac{[1,2]}{(1.1)}=0, \frac{[1,3]}{(1.2)}+\frac{[2,2]}{(1.1)}=0, \text { etc } \ldots \ldots \ldots(
$$

Hence we can at once read off the truth of the lemma for the case $h=1$. The curve lying on $\Omega$ involves that $p_{1}=0$, so that the expression for $x_{k}$ begins with the term in $s^{2}$, and the tangent prime of $\Omega$ meets the curve in two coincident points at the point considered. While, if the tangent prime meets the curve in three coincident points, that is, if $p_{2}=0$, then from the equation

$$
\frac{[1,1]}{(1.1)}+\frac{p_{2}}{2 !}=0
$$

we have $[1,1]=0$, and hence the condition for the tangent line of 
the curve to lie on $\Omega$ is satisfied for the particular value $s=0$, that is at the point of the curve which is under consideration.

The truth of the lemma for $h=2$ is also clear. If the conditions for the curve, and its tangents, to lie on $\Omega$, are satisfied, the comparison of the conditions (I) and (II) leads to

$$
p_{1}=0, p_{2}=0, p_{3}=0,
$$

so that the tangent prime of $\Omega$ at the point, $x_{k}=0$, meets the curve in four points coincident at this point. While, if the tangent prime meets the curve at this point in five coincident points, that is, if also $p_{4}=0$, then it follows from (I) and (II) that

$$
[1,3]=0 \text { and }[2,2]=0 \text {. }
$$

Now the condition that the osculating plane at the point considered should lie on $\Omega$ is that the result of substitution in the equation of the quadric $\Omega$ of the values of $d^{2} x / d s^{2}, d^{2} y / d s^{2}$, for $x, y$, say of

$$
x^{\prime \prime}=\frac{a_{2}}{0 !}+\frac{a_{3}}{1 !} s+\frac{a_{4}}{2 !} s^{2}+\ldots, y^{\prime \prime}=\frac{b_{2}}{0 !}+\frac{b_{3}}{1 !} s+\frac{b_{4}}{2 !} s^{2}+\ldots
$$

should vanish for $s=0$. This requires, however, only that $[2,2]=0$, which is one of the consequences deduced above from $p_{4}=0$.

The argument can be continued in this way; and the result is as stated. 